\title{
Choice cuts?
}

\section{$\Rightarrow$ STEM CELLS}

Developmental origin of a bipotential myocardial and smooth muscle cell precursor in the mammalian heart. Wu, S. M. et al. Cell 22 Nov 2006 (doi: 10.1016/j.cell.2006.10.028)

Multipotent embryonic Is/ $1^{+}$progenitor cells lead to cardiac, smooth muscle, and endothelial cell diversification.

Moretti, A. et al. Cell 22 Nov 2006 (doi: 10.1016/j.cell.2006.10.029)

Cardiac disorders represent one field in which there is currently great interest in the potential of therapeutic strategies based on stem cells, but the identification of the optimal cell types for such strategies is a major challenge. The endothelial, cardiac and smooth muscle

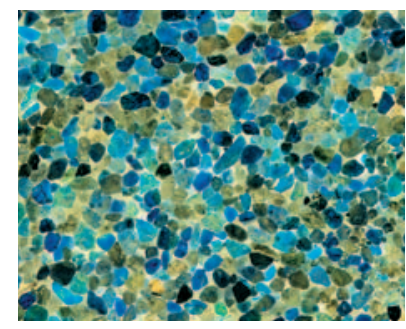
cells needed for cardiogenesis have been thought to arise from distinct embryonic precursors. However, these two papers in Cell report multipotent cardiovascular progenitor cells that are capable of developing into two and three of these cell types, respectively, which might be valuable in therapeutic cardiovascular tissue regeneration in disorders such as heart failure.

\section{$\Rightarrow$ NANOTECHNOLOGY}

Rare earth nanoparticles prevent retinal degeneration induced by intracellular peroxides.

\section{Chen, J. et al. Nature Nanotechnol. 1, 142-150 (2006)}

Reactive oxygen intermediates (ROIs) are thought to be important in the pathogenesis of retinal degeneration. Chen et al. tested their hypothesis that engineered cerium oxide nanoparticles that scavenge ROls might be able to do so within retinal neurons; their data show that these particles prevent increases in intracellular ROI concentrations in vitro and loss of vision due to light-induced degeneration of photoreceptor cells in albino rats. Such particles might therefore represent a new type of therapeutic strategy for various retinal diseases, and also other disorders in which ROls are involved.

\section{$\Rightarrow$ PARKINSON'S DISEASE}

Activation of tyrosine kinase receptor signaling pathway by rasagiline facilitates neurorescue and restoration of nigrostriatal dopamine neurons in MPTP-induced parkinsonism.

Sagi, Y. et al. Neurobiol. Dis. 25, 35-44 (2007)

Developing agents that have the potential to modify or reverse the pathogenesis of Parkinson's disease, rather than just target the symptoms, is a major challenge. Sagi and colleagues investigated the effects of rasagiline - an approved monoamine oxidase (MAO)-B inhibitor that is known to have neuroprotective activities unrelated to its ability to inhibit MAO-B - in the standard MPTP mouse model of Parkinson's disease. They show that rasagiline has neurorescue/ neurotrophic activity, and provide several lines of evidence that a mechanism involving induction of neurotrophic factors and activation of the Ras-PI3K-Akt survival pathway is important in this activity.
Evidence for the key role of amyloid- $\beta$ (A $\beta$ ) in Alzheimer's disease has led to considerable interest in potential therapeutic strategies targeting two enzymes $-\beta$ - and $\gamma$-secretase - that produce $A \beta$ through sequential cleavage of the $A \beta$ precursor protein. Three recent papers report findings that could have significant implications for these strategies.

In the first study, reported in Nature Medicine, $\mathrm{Ni}$ and colleagues sought to investigate how the pathogenesis of Alzheimer's disease is influenced by environmental factors such as stress, which is mediated by receptors including the $\beta_{2}$-adrenoceptor $\left(\beta_{2} A R\right)$. Using cell-based assays, they found that $\beta_{2} A R$-activation increased secretion of $A \beta$, which was blocked by pretreatment with a $\gamma$-secretase inhibitor. Subsequent experiments using an assay of the activity of $\gamma$-secretase indicated that the $\beta_{2}$ AR-agonist-induced increase in A $\beta$ secretion was due to increased $\gamma$-secretase activity.

Next, the authors probed the mechanism for enhanced $\gamma$-secretase activity. After finding that activity enhancement was independent of cAMP signalling, they discovered that endocytosis inhibitors or small-interfering RNA against clathrin - a protein involved in the formation of endcytotic vesicles abolished $\beta_{2} A R$-induced $\gamma$-secretase activity. So, $\beta_{2} A R$-induced enhancement of $\gamma$-secretase activity is dependent on clathrin-mediated endocytosis. Further experiments revealed that the $\beta_{2} A R$ directly associates with presenilin $1-$ the catalytic subunit of $\gamma$-secretase - which was trafficked to late endosomes and lysosomes where $A \beta$ production was elevated.

Finally, Ni et al. studied the effects of $\beta_{2} A R$ activation in rodents. Administration of either noradrenaline or a selective $\beta_{2} A R$ agonist enhanced $\gamma$-secretase activity and hippocampal A $\beta$ levels in rats. Mice with cerebral amyloid plaques chronically treated with a selective $\beta_{2} A R$ agonist had increased plaques, whereas mice treated with a selective $\beta_{2} A R$ antagonist had reduced plaque levels.

Together, these results suggest a mechanism for the pathological role of stress in Alzheimer's disease, and indicate that $\beta A R$ antagonists - which have long been widely used in cardiovascular disease therapy - might have therapeutic potential for Alzheimer's disease. This idea is supported by a recent study suggesting that the use of $\beta A R$ antagonists correlates with a decreased incidence of Alzheimer's disease.

The other two papers, in Science and Nature Neuroscience, focus on the $\beta$-secretase enzyme, which in recent years has been viewed by some as a more promising target for reducing the pathological formation of $A \beta$ than $\gamma$-secretase, in part owing to the role of $\gamma$-secretase in normal physiological processes. However, the physiological role of $\beta$-secretase has not been clear.

To investigate this role, both Willem et al. and Hu et al. used $\beta$-secretasenull (Bace1-null) mice. Lack of Bace1 resulted in the accumulation of unprocessed neuregulin 1 , which is required for glial cell development and myelination. Together, their results indicate that $\beta$-secretase has a crucial role in the myelination of peripheral and central nerves during development. Although it remains to be determined whether the influence of $\beta$-secretase is important in the maintenance of the mature myelin sheath, these studies suggest that $\beta$-secretase inhibition for Alzheimer's disease should be approached with caution.

Charlotte Harrison

ORIGINAL RESEARCH PAPERS Ni, Y. et al. Activation of $\beta$-adrenergic receptor stimulates $\gamma$-secretase activity and accelerates amyloid plaque formation. Nature Med. doi:10.1038/nm1485 (2006) Willem, M. et al. Control of peripheral nerve myelination by the $\beta$-secretase BACE1. Science 314, 664-666 (2006) | Hu, X. et al. Bace1 modulates myelination in the central and peripheral nervous system. Nature Neurosci. 9, 1520-1525 (2006) 